\title{
Chondrogenic potential of injectable $\kappa$-carrageenan hydrogel with encapsulated adipose stem cells for cartilage tissue-engineering applications
}

\author{
Elena G. Popa ${ }^{1,2}$, Sofia G. Caridade ${ }^{1,2}$, João F. Mano ${ }^{1,2}$, Rui L. Reis ${ }^{1,2}$ and Manuela E. Gomes ${ }^{1,2 *}$ \\ ${ }^{1} 3$ Bs Research Group - Biomaterials, Biodegradables and Biomimetics, University of Minho, Guimarães, Portugal \\ ${ }^{2}$ ICVS/3Bs - PT Government Associate Laboratory, Braga/Guimarães, Portugal
}

\begin{abstract}
Due to the limited self-repair capacity of cartilage, regenerative medicine therapies for the treatment of cartilage defects must use a significant amount of cells, preferably applied using a hydrogel system that can promise their delivery and functionality at the specific site. This paper discusses the potential use of $\kappa$-carrageenan hydrogels for the delivery of stem cells obtained from adipose tissue in the treatment of cartilage tissue defects. The developed hydrogels were produced by an ionotropic gelation method and human adipose stem cells (hASCs) were encapsulated in $1.5 \% \mathrm{w} / \mathrm{v}$ K-carrageenan solution at a cell density of $5 \times 10^{6} \mathrm{cells} / \mathrm{ml}$. The results from the analysis of the cell-encapsulating hydrogels, cultured for up to 21 days, indicated that $\kappa$-carrageenan hydrogels support the viability, proliferation and chondrogenic differentiation of hASCs. Additionally, the mechanical analysis demonstrated an increase in stiffness and viscoelastic properties of $\kappa$-carrageenan gels with their encapsulated cells with increasing time in culture with chondrogenic medium. These results allowed the conclusion that $\kappa$-carrageenan exhibits properties that enable the in vitro functionality of encapsulated hASCs and thus may provide the basis for new successful approaches for the treatment of cartilage defects. Copyright (c) 2013 John Wiley \& Sons, Ltd.
\end{abstract}

Received 10 October 2012; Accepted 14 November 2012

Keywords adipose-derived stem cells; cartilage; chondrogenic differentiation; hydrogels; $\kappa$-carrageenan; mechanical properties

\section{Introduction}

The need for tissue-engineered cartilage constructs is immense and of great clinical significance, because no existing medication or surgical procedures substantially promotes the healing process of articular cartilage. Compared to other connective tissues, cartilage is an avascular, aneural tissue, consisting of relatively few cells, proteoglycans and proteins, and thus, when damaged due to a degenerative disease or trauma, the functional and the metabolic properties of the original hyaline tissue will hardly ever be restored (Redman et al., 2005). Cartilage tissue-engineering approaches, adopting the delivery of an appropriate type

*Correspondence to: M. E. Gomes, 3Bs Research Group Biomaterials, Biodegradables and Biomimetics, University of Minho, Headquarters of the European Institute of Excellence on Tissue Engineering and Regenerative Medicine, Guimarães 4806-909, Portugal.E-mail: megomes@dep.uminho.pt and amount of cells, with or without signalling factors, offer considerable promise as regeneration strategies (Tuli et al., 2003; Nesic et al., 2006). However, for further improvement, minimally invasive approaches and innovative cellcarrier concepts should be refined, using the right biomaterials and cell sources (Sittinger et al., 2004). Stem cells are the best chance for human cartilage regeneration, given their ability to differentiate in vitro in different lineages, availability and expansion (Vallee et al., 2009). In particular, adipose tissue has generated significant interest in cartilage tissue engineering as an abundant source of multipotent progenitor cells, which are easily acquired at high yields and can be obtained from the patient by minimally invasive procedures, such as liposuction (Erickson et al., 2002; Augst et al., 2006; Tapp et al., 2009). Hydrogels are the basis of cell-encapsulation systems, one of the most promising approaches for the delivery of cells and therapeutic agents to the site of interest (Jen et al., 1996; Drury and Mooney, 2003; Slaughter et al., 2009). Different hydrogels 
have previously been studied for biomedical applications, such as alginate and agarose (Augst et al., 2006; Varoni et al., 2012). In the present study we evaluated the potential use of $\kappa$-carrageenan hydrogel extracted from red algae as a cell carrier system. $\kappa$-Carrageenan is an ionic natural polysaccharide that displays unique properties that may provide advantageous features for application in the tissue-engineering field. Carrageenan is composed of large, highly flexible molecules which curl, forming helical structures (Bixler, 1994; Sipahigil and Dortunc, 2001), rendering them able to form a variety of different gels at room temperature and thus enabling great versatility. The fully carbohydrate-based hydrogels of alternating copolymers of $\alpha$-(1-3)-D-galactose and $\beta$-(1-4)-3,6-anhydro-Dor L-galactose (Meunier et al., 2001) are expected to be biologically compatible, biodegradable and non-toxic. $\kappa$-Carrageenan has only one negative charge per disaccharide, with a tendency to form a strong and rigid gel. Carrageenan gels in the presence of potassium ions but also has the ability to form gels under salt-free conditions, being a thermosensitive hydrogel (Lennart, 2006). The mechanism of gel formation is still under discussion; nevertheless, the first step may well be double helix stabilization when $\mathrm{K}^{+}$is the counter-ion (Kara et al., 2003). Because of the ionic nature of the polymer, the gelling and melting temperatures of $\kappa$-carrageenan are almost solely dependent on the concentration of potassium ions (Mangione et al., 2005). Thermoreversible gels, such as $\kappa$-carrageenan, melt at elevated temperatures, and lowering the temperature results in the gelation of the biopolymer. The temperature-induced gelation allows for the easy formation of gels in different shapes. The negative charge permits ionic interactions with molecules such as water or protein and allows a large osmotic swelling pressure. To some extent, $\kappa$-carrageenan resembles naturally occurring glycosaminoglycans, owing to their backbone composition of sulphated disaccharides (Velleman, 1999). At present there is little information about the potential use of $\kappa$-carrageenan as a new natural material in biomedical applications. Nevertheless, the results published to date suggest that $\kappa$-carrageenan hydrogels exhibit comparable biological behaviour to, but higher mechanical properties than, similar hydrogels extensively used for tissue-engineering purposes (Awad et al., 2004; Bosnakovski et al., 2006; Kisiday et al., 2008). The objective of this study was to develop $\kappa$-carrageenan hydrogel aimed at application in cartilage tissue engineering. Therefore, the swelling and cytotoxicity of the hydrogels were studied, as well as their ability to sustain the proliferation and chondrogenic differentiation of hASCs. Furthermore, the mechanical properties of hydrogels with encapsulated hASCs after different times in culture were also evaluated. The results obtained demonstrated that $\kappa$-carrageenan hydrogels are non-cytotoxic and support the proliferation and chondrogenic differentiation of encapsulated hASCs. Additionally, the mechanical properties of the cell-loaded hydrogels are maintained and even increased with increasing culture time, suggesting that the proposed systems provides a promising alternative to current approaches for cartilage tissue engineering.

\section{Materials and methods}

\section{1. $\kappa$-Carrageenan hydrogel preparation}

An aqueous solution was prepared by dissolving the $\kappa$-carrageenan powder (Sigma Fluka) in distilled water, and heating at $60^{\circ} \mathrm{C}$ while stirring constantly for $2 \mathrm{~h}$ until complete and homogeneous dispersion of the material was obtained. Previous to use, the $\kappa$-carrageenan solution was autoclaved for $30 \mathrm{~min}$ at $120^{\circ} \mathrm{C}$ and prepared just before use. $\kappa$-Carrageenan hydrogel samples were produced using the sterilized polymeric solution with a final concentration of $1.5 \% \mathrm{w} / \mathrm{v}$ and $5 \% \mathrm{w} / \mathrm{v}$ potassium chloride $(\mathrm{KCl}$; Sigma) as a crosslinker reagent. The samples were shaped in the form of discs, using cylindrical moulds, and a complete gelation carried out for $2-5 \mathrm{~min}$ at room temperature to form a solid gel. After gelation, the moulds' ends were cut and the cylindrical hydrogels pushed out and immersed in $\mathrm{KCl}$ for $15-30 \mathrm{~min}$ in order to stabilize the three-dimensional (3D) structure. Afterwards, the gels were washed with phosphate-buffered saline (PBS; Sigma) to remove the excess $\mathrm{KCl}$ present in the materials. Sample discs were then sliced off the hydrogel cylinders, using a thin sterile blade; disc dimensions were diameter $5 \pm 0.01 \times$ height $2.5 \pm 0.46 \mathrm{~mm}$. These samples were used for evaluating the swelling kinetics and cytotoxicity of the hydrogel.

\section{2. $\kappa$-Carrageenan hydrogel physical characterization}

\subsubsection{Swelling kinetics}

The swelling kinetics of the developed hydrogels was studied in a PBS solution (different $\mathrm{pH}$ values were tested) and in culture medium [Dulbecco's modified Eagle's medium (DMEM), supplemented with fetal bovine serum (FBS; Gibco]. For this purpose, a weighed amount of each hydrogel formulation was immersed in each testing solution (PBS and DMEM, pH 7.4) and incubated at $37^{\circ} \mathrm{C}$ under static conditions. The influence of $\mathrm{pH}$ of the PBS solution on the water uptake of $\kappa$-carrageenan hydrogel was also analysed. The swelling ratio was calculated using Equation 1:

$$
\% \text { Equilibrium swelling ratio }(E S R)=\frac{W_{\mathrm{s}}-W_{\mathrm{d}}}{W_{\mathrm{d}}} \times 100
$$

where $W_{\mathrm{s}}$ is the weight of swollen gel after reaching equilibrium value under specified environmental conditions (PBS or DMEM) and $W_{\mathrm{d}}$ is the weight of the dried gel. To measure $W_{s}$, the swollen hydrogels were removed from the PBS or DMEM and immediately weighed with a microbalance after the excess of water lying on the surfaces was absorbed with a filter paper. Three samples were used for each testing condition $(n=3)$. 


\section{3. $\kappa$-Carrageenan hydrogel biological evaluation}

\subsubsection{Cytotoxicity screening}

The cytotoxicity of the leachable hydrogels was evaluated using L929 mouse fibroblast cells [European Collection of Cell Cultures (ECACC), UK], as described elsewhere (Gomes et al., 2001). Extracts of the study materials were prepared and placed in contact with a L929 cells monolayer. Briefly, the extracts were obtained after $24 \mathrm{~h}$ incubation of the hydrogels in complete culture medium at $37^{\circ} \mathrm{C}$ and under constant agitation $(60 \mathrm{rpm})$. The ratio of the hydrogel to the extract fluid was $3 \mathrm{~cm}^{2} / \mathrm{ml}$ and the $\mathrm{L} 929$ cell-seeding density was $4 \times 10^{3}$ cells/well plate. In all cytotoxicity tests performed, latex rubber was used as the positive control for cell death and standard tissue culture polystyrene was used as a negative control. The objective of the extraction test was to evaluate changes in cell morphology and growth inhibition, whereas the MTS (CellTiter $96^{\circledR}$ Aqueous One Solution Cell Proliferation Assay, G3580, Promega) test determines whether cells are metabolically active (Salgado et al., 2004). The optical density (OD) was read at $490 \mathrm{~nm}$ on a multiwell microplate reader (Synergie Ht Izasa, Bio-Tek Instruments) and all cytotoxicity screening tests used six replicates.

\subsubsection{Human adipose-derived stem cells isolation and expansion}

Human liposuction aspirate samples were obtained following informed consent from donors undergoing lipoaspiration procedures under a protocol established with the Department of Plastic Surgery of the Hospital da Prelada, Porto, and approved by the local Ethical Committee. All the samples were processed within $24 \mathrm{~h}$ after collection. Human adipose-derived stem cells (hASCs) were enzymatically isolated as previously described (Rada et al., 2010). Briefly, the adipose tissue samples were digested with $0.2 \%$ collagenase type II (Sigma) in PBS for $45 \mathrm{~min}$ at $37^{\circ} \mathrm{C}$ under gentle stirring. The digested tissue was filtered with a $100 \mu \mathrm{m}$ filter mesh (Sigma) centrifuged at $1200 \mathrm{rpm}$ for $10 \mathrm{~min}$ at $20^{\circ} \mathrm{C}$, and the cell suspension solution washed for $5 \mathrm{~min}$ with lysis buffer to remove erythrocytes. The cells were again centrifuged and the supernatant removed and resuspended in $\alpha$-minimal essential medium ( $\alpha$-MEM; Gibco) with 10\% FBS (Gibco; heat-inactivated), 1\% antibiotic-antimycotic (Invitrogen) and sodium bicarbonate $\left(\mathrm{NaHCO}_{3}\right.$; Sigma). Human ASCs were plated at a density of $3.5 \times 10^{3}$ cells $/ \mathrm{cm}^{2}$ and incubated at $37^{\circ} \mathrm{C}$ in a humidified atmosphere of $5 \% \mathrm{CO}_{2}$. The unattached cells were removed after 2-3 days with repeated PBS washings. The adherent hASCs were cultured with medium changes every 3 days. Confluent cultures were passaged with $0.05 \%$ trypsin (Invitrogen). The enzymatic treatment was quenched in the presence of FBS and cell counts were done using a haemocytometer. Only passage 3 (P3) hASCs were used in the experiments.
For the cell culture experiments, adipose-derived stem cells were isolated from one patient (a women aged 31 years) and cryopreserved at passage 1 , to avoid patient cells variability. Afterwards, the cells were expanded and used until P3, performing three independent experiments using the same batch of cells, with the same passage. The phenotype of hASCs has been extensively investigated in previous studies, using tissue sample from the same anatomical site (subcutaneous), harvested with a similar technique (such as lipoaspiration) and isolated following similar procedures (digestion with collagenase) by Zuk et al. (2002) and Gimble et al. (2007) and also by our research group (Carvalho et al., 2011a, 2011b; Rada et al., 2011).

\subsubsection{Encapsulation of hASCs in $\kappa$-carrageenan hydrogels and in vitro cell culture}

$\kappa$-Carrageenan aqueous dispersions were prepared just before use by dissolving the biopolymer in distilled water and then sterilizing, as described in previous section. Human ASCs were detached by trypsin and centrifuged at $200 \times g$ for $7 \mathrm{~min}$. The cells were resuspended in sterile PBS solution, counted using a haemocytometer and finally centrifuged. The supernatant was discarded and $\kappa$-carrageenan $1.5 \% \mathrm{w} / \mathrm{v}$ solution was added to the cells to obtain a final concentration of $5 \times 10^{6} \mathrm{cells} / \mathrm{ml}$. The mixture was resuspended for complete homogenization of the cells within the matrix. Hydrogel samples containing human ASCs were prepared using sterile cylindrical moulds and allowing to rest at room temperature for 1-2 $\mathrm{min}$ to form a solid gel. Discs of $5 \pm 0.01 \mathrm{~mm}$ diameter and $2.5 \pm 0.46 \mathrm{~mm}$ height were cut using a sterile blade. The discs with encapsulated cells were cultured in either basal or chondrogenic differentiation medium for 1, 7, 14 and 21 days, with the medium replaced every 3-4 days. Additional controls consisted of $\kappa$-carrageenan hydrogel samples without cells, kept under the same culture conditions for the selected time periods. The chondrogenic differentiation medium was composed of low-glucose DMEM (Sigma) supplemented with 10\% FBS (Gibco), 1\% antibiotic-antimycotic (Gibco), ITS +1 Liquid Media Supplement (I2521; insulin-transferrin-selenium liquid media supplement, Sigma), $17 \mathrm{mmL}$-ascorbic acid (Sigma), $0.1 \mathrm{~m}$ sodium pyruvate (Sigma), $35 \mathrm{mmL}$-proline (Sigma), $1 \mathrm{~mm}$ dexamethasone (Sigma) and $10 \mathrm{ng} / \mathrm{ml}$ human transforming growth factor- $\beta 1$ (TGF $\beta 1$; eBioscience).

\section{4. $\kappa$-Carrageenan hydrogel biological characterization}

\subsubsection{Cell viability and proliferation assessment}

At the end of each time point of the study, the $\kappa$-carrageenan samples with encapsulated hASCs were collected from the culture plates and incubated in a Calcein AM (Invitrogen) solution of $1 / 1000$ in culture medium for $15-30 \mathrm{~min}$ at $37^{\circ} \mathrm{C}$ and afterwards washed in sterile PBS. The stained samples were placed on a microscope slide and observed 
under fluorescent microscopy (reflected/transmitted light microscope; Zeiss). The proliferation of the hASCs encapsulated in the $\kappa$-carrageenan hydrogels was assessed using a fluorimetric double-strand DNA quantification kit (PicoGreen, Molecular Probes). For this purpose, samples collected at 1, 7, 14 and 21 days of culture were washed in PBS and then transferred into $1.5 \mathrm{ml}$ microtubes containing $1 \mathrm{ml}$ ultrapure water. Prior to dsDNA quantification, hASChydrogel constructs and the sample controls (hydrogel samples without cells) were sonicated for $15 \mathrm{~min}$ to release all DNA from the hydrogel. Samples and standards $(0-2 \mu \mathrm{g} / \mathrm{ml})$ were prepared and mixed with a PicoGreen solution in a 200:1 ratio and added to a 96-well opaque white plate. For each study material and standard, three samples were used for DNA assays, and triplicates of each sample were measured; the procedure followed can be found elsewhere (Labarca and Paigen, 1980) and was based on the manufacturer's instructions. The plate was incubated for $10 \mathrm{~min}$ in the dark and fluorescence was measured on a microplate ELISA reader (Bio-Tek, Synergie HT) with an excitation of $485 / 20 \mathrm{~nm}$ and an emission of 528/20 nm. A standard curve was created, and sample DNA values were read from the standard graph.

\subsubsection{Histological analysis}

Samples were collected at the end of the experiment (21 days of culture) fixed, dehydrated with a Spin Tissue Processor (Microm STP120 Inopat) and embedded in paraffin using an embedding centre (Microm EC350-1/ EC350-2 Inopat). Sections were cut at $4 \mu \mathrm{m}$ with a microtome (Microm HM355S Inopat) and placed on microscopy slides. Alcian blue (Sigma), safranin O (Sigma), toluidine blue (Sigma) and haematoxylin and eosin (H\&E; Sigma) stainings were performed using the Automatic Stainer equipment (Microm HMS740 Inopat). Alcian blue, safranin $\mathrm{O}$ and toluidine blue were used to evaluate cartilage ECM components (glycosaminoglycans) deposition. Alcian blue staining was performed by rinsing the sections in 3\% acetic acid (Sigma) and incubating them in 1\% alcian blue solution for $30 \mathrm{~min}$. After that, the stain was poured off and the sections counterstained with aqueous neutral red (Sigma) for $1 \mathrm{~min}$, then dehydrated. Safranin O staining consisted of staining the sections with Weigert's iron haematoxylin working solution for $7 \mathrm{~min}$, fast green (Sigma) for $5 \mathrm{~min}$ and $0.1 \%$ safranin $\mathrm{O}$ for $5 \mathrm{~min}$. The sections were washed after each staining step, left to dry in air and then rinsed in absolute alcohol. Toluidine blue staining solution was prepared by dissolving $1 \%$ toluidine blue in distilled water containing $0.5 \mathrm{~g}$ sodium borate (Riedelde-Haën), followed by filtering, and the sections were dipped in for 2-3s. For H\&E staining, after hydration the sample sections were coloured with Papanicolaou Harris haematoxylin (Bio-optica) for $3 \mathrm{~min}$, washed in running tap water and afterwards a blue stain enhancement was performed by immersion in $0.5 \%$ ammonia (Sigma) for $5-10 \mathrm{~s}$. The sections were then washed in running tap water and stained in eosin-Y (Bio-optica) for $30 \mathrm{~s}$. Finally all slides were dehydrated by immersion in a series of alcohols (30-100\%). The final step for all stainings was immersion in the clearing agent Histoclear $^{\circledR}$ (National Diagnostics) or xylene substitute for 1-2 min, then mounting using Microscopy Entellan ${ }^{\circledR}$ (Merck) for later observation. The stained sections were observed under a light microscope (reflected/transmitted light microscope; Zeiss).

\subsubsection{Immunohistochemical analysis}

Sections of samples corresponding to all experimental conditions were obtained as described above. Before removing the paraffin the slides were warmed, and antigen retrieval was performed for $20 \mathrm{~min}$ at $95^{\circ} \mathrm{C}$ using $10 \mathrm{~mm}$ citrate buffer. The sections were washed in PBS for $10-15 \mathrm{~min}$ and endogenous peroxidase activity was quenched with $3 \%$ hydrogen peroxide (Sigma) in 50\% methanol/tap water for $5 \mathrm{~min}$. Afterwards, the samples were washed with PBS and blocked with 3\% bovine serum albumin (BSA; Sigma) for $1 \mathrm{~h}$ to avoid non-specific staining. The sections were further incubated with primary antibodies [collagen types I and II; mouse antitype II collagen (MAB1330) and mouse antitype I collagen (MAB3391); Chemicon) overnight at $4{ }^{\circ} \mathrm{C}$ in a humidified atmosphere. Then the slides were washed with PBS for $10 \mathrm{~min}$ and incubated with secondary antibody from a Vectastain Elite ABC Kit (Vector Laboratories, Peterborough, UK) for $1 \mathrm{~h}$ at room temperature, again in a humidified atmosphere. The remaining protocol was performed according to that described in the in the Vector DAB Kit (Vector Laboratories). The slides were washed in water for $5 \mathrm{~min}$ and then counterstained with haematoxylin for nuclei visualization, and finally mounted. Controls were performed using normal horse serum replacing the primary antibodies, which was also included in the kit. The samples were visualized under a light microscope and images obtained using a camera (Axion MRc5, Zeiss).

\subsubsection{RNA isolation and real-time quantitative $R T-P C R$}

Real-time qRT-PCR analysis was used to assess the expression profile of typical markers for chondrogenic differentiation, namely Sox9, Aggrecan, Collagen $X$, Collagen type I and Collagen type II, and thereby to evaluate the ability of hASCs to undergo differentiation when encapsulated in $\kappa$-carrageenan and cultured with either basal or chondrogenic medium. For this purpose, total RNA was extracted from cell-hydrogel constructs using TRI Reagent ${ }^{\circledR}$ RNA Isolation Reagent (Sigma) according to the technical data sheet provided. Briefly, three samples of each condition were collected at defined time periods, washed twice with PBS, added to $800 \mu \mathrm{l}$ TRI Reagent and vigorously mixed for $10 \mathrm{~s}$, then stored at $-80^{\circ} \mathrm{C}$ until the analysis was performed. At this point, $160 \mu$ l chloroform was added and the samples incubated on ice for $15 \mathrm{~min}$ and centrifuged at $13000 \mathrm{rpm}$ for $15 \mathrm{~min}$ at $4^{\circ} \mathrm{C}$ to establish a three-phase composition in the tube. The aqueous phase was collected into new, clean, prechilled 
tubes, where $400 \mu \mathrm{l}$ ice-cold isopropanol was added, and the samples were incubated at $-20^{\circ} \mathrm{C}$ overnight. The samples were then centrifuged at $13000 \mathrm{rpm}$ for $15 \mathrm{~min}$ at $4{ }^{\circ} \mathrm{C}$, the supernatant discarded and the pellet washed with $70 \%$ ethanol. After a final centrifugation, the samples were allowed to dry in air and suspended in ultrapure water for posterior analysis. Each pellet was dissolved in $15 \mu \mathrm{l}$ RNase-free water and kept at $-80^{\circ} \mathrm{C}$ until use. The amount of isolated RNA and $\mathrm{A}_{260 / 280} \mathrm{~nm}$ ratio were quantified using a Nanodrop ND-1000 spectrophotometer (Bonsai 06/2008 NanoDrop Technologies, Wilmington, DE, USA). After these determinations, $2 \mu \mathrm{g}$ RNA of each sample was reverse-transcribed using a qScript ${ }^{\mathrm{TM}} \mathrm{cDNA}$ Synthesis Kit (Quanta Biosciences) in a $40 \mu \mathrm{l}$ reaction, using a MJ Mini ${ }^{\mathrm{TM}}$ Personal Thermal Cycler (Bio-Rad Laboratories) machine. Real-Time qRT-PCR was performed to detect amplification variations, using $\operatorname{PerfeCTa}^{\circledR}$ SYBR $^{\circledR}$ Green FastMix ${ }^{\circledR}$ (Quanta Biosciences) on an Eppendorf Mastercycler ${ }^{\circledR}$ ep realplex gradient $S$ machine. The analysis of the results was performed with realplex software (Eppendorf Mastercycler, Applied Biosystems). The reaction composition was as follows: $10 \mu \mathrm{l}$ SYBR Green PCR FastMix, $2.5 \mu \mathrm{l}$ each forward and reverse primer (see Table 1 ) and $5 \mu \mathrm{l}$ diluted template with RNase-free water. The number of cycles and annealing temperature were selected according to the manufacturer's instructions. All the primer sequences were generated using Primer3 software v 0.4.0 and acquired from Eurofins MWG Operon (Ebersberg). More details can be found in Table 1. Each gene was processed in triplicate. Human ASCs encapsulated in $\kappa$-carrageenan hydrogel cultured in basal medium were used as control cell sample. In each sample the mRNA level expression of each gene was normalized to the average expression of GAPDH value. The relative gene expression quantification was performed using the $2^{-\triangle C \mathrm{~T}}$ and $2^{-\triangle \triangle T}$ methods (Livak and Schmittgen, 2001). Three samples of each material/conditions under study were analysed.

\subsubsection{Mechanical properties of $\kappa$-carrageenan hydrogels with encapsulated hASCs}

Dynamic mechanical analysis (DMA; Tritec 2000 Triton) was conducted to characterize the mechanical properties, under compression loading, of $\kappa$-carrageenan hydrogel samples and hydrogels samples with encapsulated hASCs, after culture in either chondrogenic or basal medium for different time points. The samples were prepared, as described above, into discs of $8 \pm 0.01 \mathrm{~mm}$ diameter and $2.5 \pm 0.38 \mathrm{~mm}$ height. The study samples $(n=5)$ were subjected to compression cycles of increasing frequency in the range $0.1-10 \mathrm{~Hz}$, with constant amplitude displacements of $0.1 \mathrm{~mm}$, using DMA equipment (Triton Technology, Nottinghamshire, UK). Experiments were performed under simulated physiological conditions at $37^{\circ} \mathrm{C}$ in PBS medium. The frequency scans, with acquisitions of 15 points/decade, were performed at $37^{\circ} \mathrm{C}$ with a heating rate of $2{ }^{\circ} \mathrm{C} / \mathrm{min}$. The mechanical analysis results were presented in terms of two main parameters: storage modulus ( $E^{\prime}$; the in-phase, elastic component) and loss modulus ( $E^{\prime \prime}$; the out-of-phase, viscous component). The values for the compression modulus were collected at a frequency of $0.1-10 \mathrm{~Hz}$ along the 21 days of culture, and damping factor was expressed for samples cultured in chondrogenic medium.

\subsection{Statistical analysis}

Data obtained from DNA and real-time qRT-PCR analysis are presented as mean \pm standard deviations (SD; $n=3$ ). First, a Shapiro-Wilk test was used to ascertain the data normality. DMA data were first inspected with an F-test for treatments, to determine equality of variance (Liski, 2009). The results indicated that at the 0.05 level, the data were significantly drawn from a normally distributed population, and one-way ANOVA followed by Tukey test was used to determine significant differences between groups and conditions, using with OriginPro 8 program.

\section{Results and discussion}

\subsection{Swelling kinetics of $\kappa$-carrageenan hydrogel}

The $\kappa$-carrageenan hydrogels were obtained by a mild crosslinking reaction with $\mathrm{K}^{+}$ions at a stable physiological $\mathrm{pH}$. The influence of phosphate buffer solution $\mathrm{pH}$ on the swelling properties of $\kappa$-carrageenan hydrogels was analysed, determining the equilibrium swelling at $\mathrm{pH} 1,4,7.4$ and 11 as a function of time (Figure 1). These results showed that swelling increases with time, reaching a constant maximum

Table 1. List of genes under evaluation, primers and annealing temperatures used in the analysis of samples corresponding to encapsulated hASCs in $\kappa$-carrageenan and cultured in vitro for different time points

\begin{tabular}{|c|c|c|c|}
\hline Target gene $^{1}$ & Forward sequence & Reverse sequence & $T_{\mathrm{m}}\left({ }^{\circ} \mathrm{C}\right)$ \\
\hline SOX9 & $5^{\prime}$-tacgactacaccgaccacca-3' & $5^{\prime}$-ttaggatcatctcggccatc-3' & 58.4 \\
\hline Aggrecan & $5^{\prime}$-tgagtcctcaagcctcctgt-3' & $5^{\prime}$-tggtctgcagcagttgattc-3' & 58.4 \\
\hline Collagen X & $5^{\prime}$-ccaggtctcgatggtcctaa-3' & $5^{\prime}$-gtcctccaactccaggatca-3' & 59.4 \\
\hline Collagen I & $5^{\prime}$-catctccccttcgtttttga-3' & $5^{\prime}$-ccaaatccgatgtttctgct-3' & 55.3 \\
\hline Collagen II & $5^{\prime}$-gacaatctggctcccaac-3' & $5^{\prime}$-acagtcttgccccacttac-3' & 56.4 \\
\hline GAPDH & $5^{\prime}$-acagtcagccgcatcttctt-3' & $5^{\prime}$-acgaccaaatccgttgactc- $3^{\prime}$ & 57.3 \\
\hline
\end{tabular}

${ }^{1}$ Real-time qRT-PCR thermal cycles correspond to the following periods: 2 min at $95^{\circ} \mathrm{C}$ (hot start), followed by $45 \mathrm{cycles}$ of $95^{\circ} \mathrm{C}$ for $30 \mathrm{~s}$ corresponding annealing temperature (noted in the table) for $30 \mathrm{~s}$ and an extension step at $68^{\circ} \mathrm{C}$ for $30 \mathrm{~s}$. A melting curve of $21 \mathrm{~min}$ and a hold step at $5^{\circ} \mathrm{C}$ was performed at the end. 


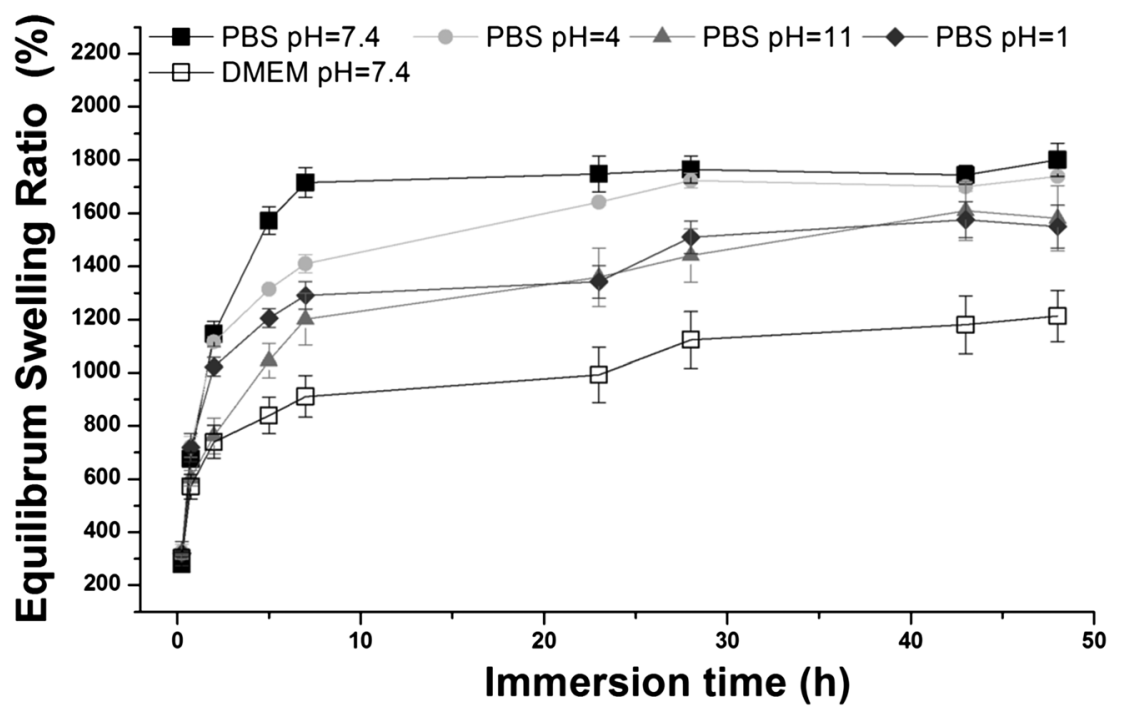

Figure 1. Equilibrium swelling ratio of $\kappa$-carrageenan hydrogels as a function of time, registered after immersion in PBS solution at different $\mathrm{pH}(1,4,7.4,11)$ and after immersion in DMEM/10\% FBS at $\mathrm{pH} 7.4$ and $37^{\circ} \mathrm{C}$. Values reported as averages $(n=3) \pm \mathrm{SD}$

value after $48 \mathrm{~h}$. In general, it was observed that the swelling ratio increased with increasing $\mathrm{pH}$ values of the $\mathrm{PBS}$ solution, except for $\mathrm{pH} 11$, which registered the lowest value for the first $28 \mathrm{~h}$ post-immersion. The amount of water absorbed during the first $7 \mathrm{~h}$ in $\mathrm{PBS}$ at $\mathrm{pH} 7.4$ registered the highest values, showing that the developed hydrogels exhibit the highest water uptake at physiological $\mathrm{pH}$. The swelling kinetics of $\kappa$-carrageenan hydrogels were also investigated when immersed in cell culture medium (DMEM, pH 7.4, at $37^{\circ} \mathrm{C}$ ) supplemented with 10\% serum (FBS) and compared with

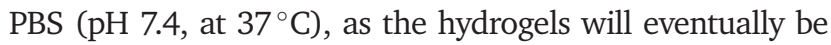
exposed to this medium during cell encapsulation and culture (Figure 1). It could be observed that the swelling ratio in DMEM/10\% FBS medium was lower than in PBS solution.

It is known that, upon implantation of a biomaterial in the body, a response typically occurs that is characterized by a decrease in the local $\mathrm{pH}$. Thus, it was thought it would be important to know the behaviour of the hydrogel at various pHs in order to predict and eventually tailor its response upon implantation, envisioning in vivo studies and possible future clinical applications. When increasing the $\mathrm{pH}$ values of the immersion solutions from 1 to values equal to the physiological $\mathrm{pH}$, a significant increase of swelling ratio leading to high water absorption was observed (Figure 1 ). The $\kappa$-carrageenan hydrogels soaked in medium at $\mathrm{pH} 11$ presented lower water uptake, revealing that the equilibrium swelling was dependent upon $\mathrm{pH}$ and the ionic composition of the immersion media. As the $\mathrm{pH}$ of the PBS solution was $>4$, the ionization of the sulphated groups of $\kappa$-carrageenan gel occurred and led to ionic repulsion. This resulted in a more hydrophilic polymer network and contributed to higher water absorption. In summary, the swelling results demonstrated that the hydrogels exhibited a pH-dependent pattern in the range of $\mathrm{pH}$ studied. Furthermore, these results support that the amount of ions present in the medium significantly affects the swelling properties of the hydrogels, as confirmed by the different results obtained upon immersion in PBS and DMEM media (Naim et al., 2004). It is important to note that ionically crosslinked $\kappa$-carrageenan loses stability over time in vitro, most probably due to an outward flux of crosslinking ions into the surrounding medium (Shoichet et al., 1996).

\subsection{Cytotoxicity assessment}

The cell behaviour of hydrogel extracts was studied as a preliminary approach to testing the in vitro toxicity of the developed $\kappa$-carrageenan hydrogels. The cytotoxicity of $\kappa$-carrageenan hydrogels was evaluated by culturing L929 cells for 1 and 3 days with extracts of the developed material. Figure 2 shows the results of cell metabolic

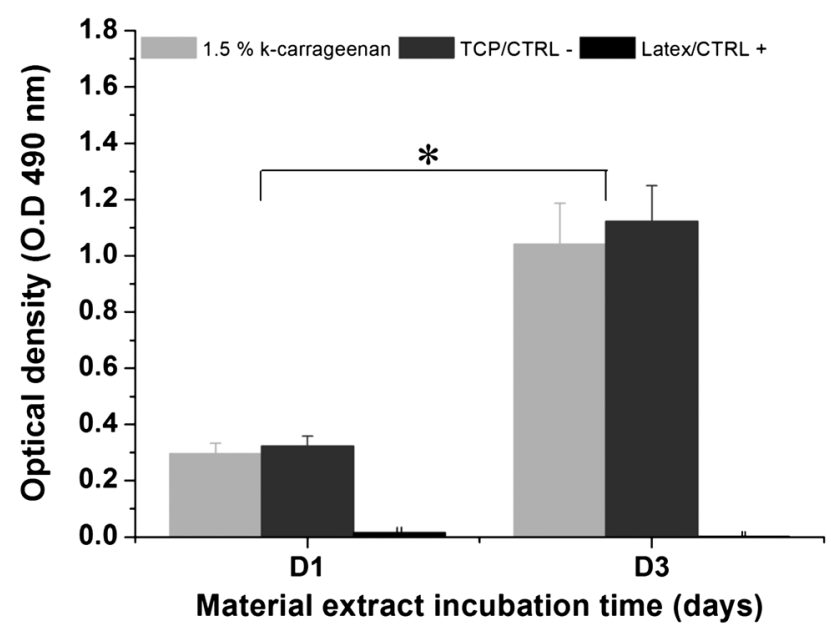

Figure 2. Graphical representation of L929 cells metabolic activity after incubation with extracts of $1.5 \% \mathrm{w} / \mathrm{v} \kappa$-carrageenan hydrogel, TCP (negative control) and latex (positive control) evaluated by MTS assay. *Significantly higher metabolic activity at day 3 as compared to values obtained at day 1 of culture. Statistical analyses were conducted using one-way ANOVA for $n=6$ and error bars represent mean \pm SD 
activity assessed by the MTS test performed with hydrogel extracts. It can be observed that the viability levels were similar to the values obtained for the negative control (TCPs), evidencing that $\kappa$-carrageenan and possible leachable products did not exert any cytotoxicity effect on L929 cells. A significant increase in cell viability was observed from day 1 to the last time point of the assay (* $p<0.05)$. The cytotoxicity assessment of $\kappa$-carrageenan extracts was carried out as a preliminary approach to assessing the potentially harmful effect of the developed hydrogels. The cytotoxicity results (Figure 2) indicated that the cells were viable in the presence of hydrogel extracts for all the time points assessed. The cells in contact with the hydrogel extracts recorded levels of metabolic activity similar to those which were in contact with culture medium, showing extremely low cytotoxicity levels, even after long exposure.

\subsection{Fluorescence staining and DNA quantification}

Fluorescence staining was conducted to analyse the viability of the cells after encapsulation/culture on $1.5 \%$ $\mathrm{w} / \mathrm{v} \kappa$-carrageenan hydrogels (Figure 3A). Calcein AM confirmed the viability of hASCs after in vitro culture, establishing that the cells were viable and homogeneously distributed inside the hydrogels. Three weeks postencapsulation, a high density of cells was observed and the cells were viable, exhibiting a round shape within the hydrogel matrix. These results confirmed that the temperature cycle used to promote the sol-gel transition of $\kappa$-carrageenan did not affect cell viability.

Complementary to the viability analysis along the course of the experiment, the cell content was quantified based on dsDNA quantification (Figure 3B). Analysis of obtained DNA values confirmed that from day 1 to day 7 the cell number slightly decreased for the two conditions, followed by a significant increase from day 14 to day 21 ( $* p<0.05$ for basal medium and $* * p<0.05$ for chondrogenic medium). These results are also in agreement with the fluorescence viability assay (Figure 3A), showing that the $\kappa$-carrageenan hydrogel enables the viability and proliferation of encapsulated hASCs.

An important aspect for hydrogels intended for use as cell carriers is how the encapsulation process affects the target cells. These hydrogels have been used to encapsulate enzymes, proteins or drugs and then release them through dissolution of the hydrogel structure (Baeza et al., 2002; Spagnuolo et al., 2005; Prakash and Martoni,

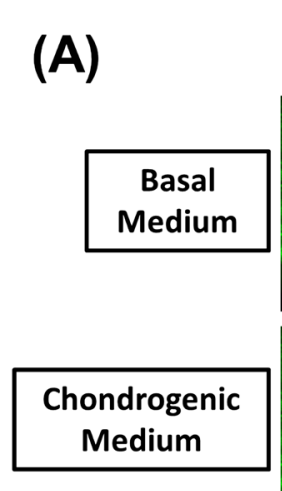

(B)
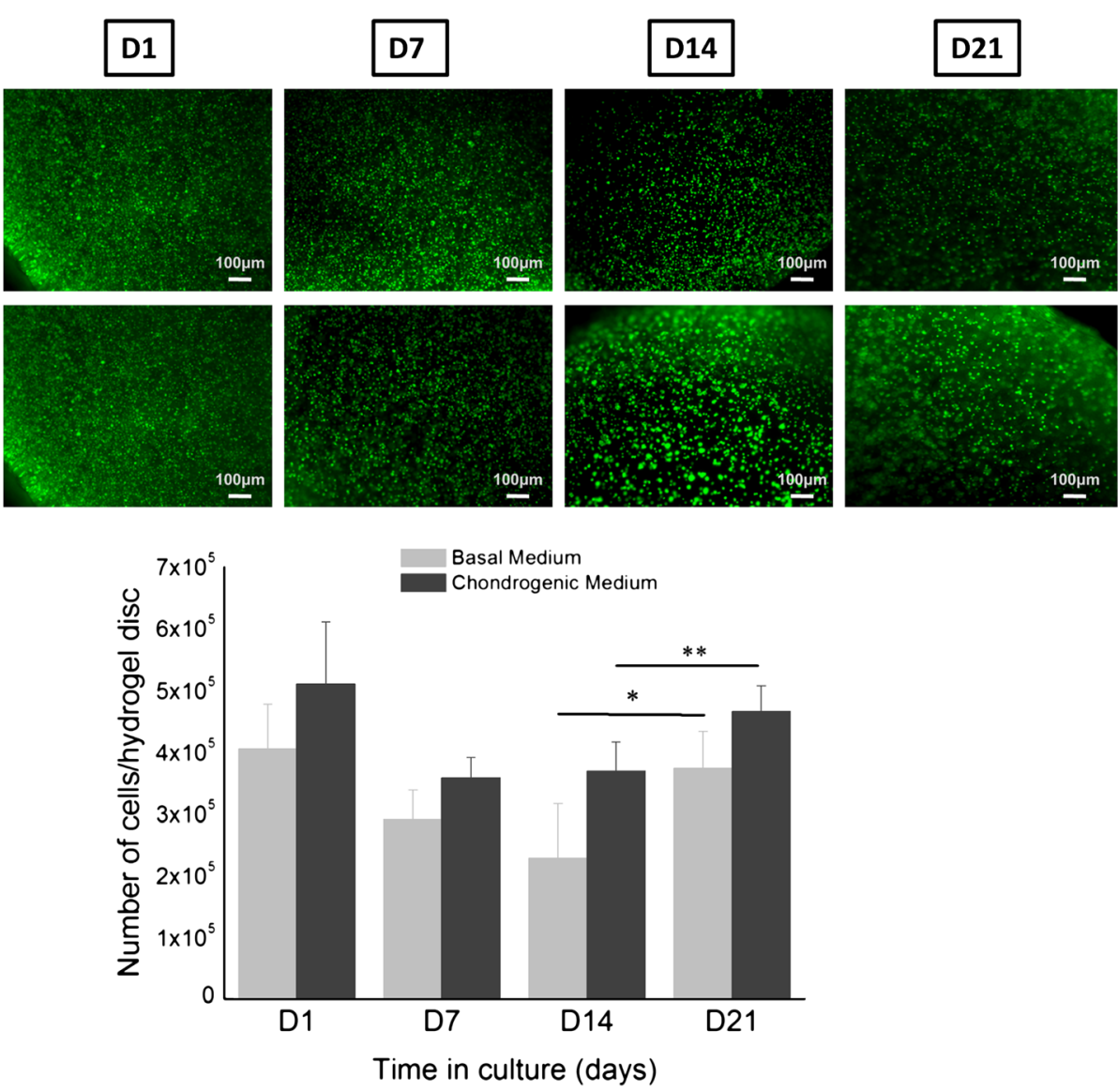

Figure 3. Viability and proliferation of human adipose stem cells (hASCs) encapsulated in $\kappa$-carrageenan hydrogels and cultured for different time points in basal and chondrogenic medium. Fluorescent images (Calcein AM staining) showing the viability and distribution of encapsulated hASCs after 21 days of culture; magnification $=\times 5$, with $100 \mu \mathrm{m}$ scale bar (A). Proliferation of hASCs cultured in basal and chondrogenic medium, results based on DNA test performed after 1, 7, 14 and 21 days of culture (B). Error bars represent mean $\pm S D$. Cell number is statistically significantly higher at 21 days than at 14 days for both basal and chondrogenic conditions $(p<0.05)$. 
2006), but the use of $\kappa$-carrageenan for cell delivery is still poorly exploited in the literature. It seemed that a significant drop in cell proliferation from the initial time could be noticed and this behaviour could be due to the fact that cells were being released from the cell-loaded hydrogel systems during culture time, owing to degradation of the hydrogels. Gelation of $\kappa$-carrageenan hydrogel was induced by $\mathrm{KCl}$ treatment, which might initially have affected cell viability, since for fast hydrogel formation a high $\mathrm{KCl}$ concentration was used and the remaining $\mathrm{KCl}$ may have influenced viability. Moreover, decreased cell viability upon cell encapsulation in hydrogels is a quite common finding, confirmed in several studies reported in literature (Awad et al., 2004; Marsich et al., 2008; Wei et al., 2008).

From the DNA assay data (Figure 3B), it was possible to confirm that until day 14 of culture the different culture conditions induced significant changes in the proliferation of hASCs. Usually stem cell proliferation decreases when the differentiation process is activated; thus, the decrease in cell content could be attributed to this characteristic. The results obtained regarding the rate of proliferation of the hASCs cells during the period when they were submitted to chondrogenic differentiation medium indicate that from day 14 to day 21 of culture, the cell proliferation rate increased significantly $(* * p<0.05)$. Furthermore, hydrogels loaded with hASCs cultured in basal medium presented significantly lower proliferation than hASCs cultured in chondrogenic differentiation medium. Similar studies using other hydrogels, such as collagen, alginate or agarose, using either BMSCs (Bosnakovski et al., 2006) or ASCs (Awad et al., 2004), showed similar cell behaviour. Together, these results indicate that $\kappa$-carrageenan enables the viability and proliferation of encapsulated human ASCs. The chemical composition of $\kappa$-carrageenan hydrogels, similar to that of the native ECM, directly affects the water content of the developed carriers, which is expected to support the phenotype of chondrocytes.

In this study hASCs were encapsulated, given that they are of particular interest for ease of harvest and their potential in therapies to promote cartilage repair, and since there are numerous studies demonstrating that cells isolated from fat tissue can undergo chondrogenic differentiation when cultured in adequate in vitro conditions (Jung et al., 2009; Guilak et al., 2010). Studies indicated that bone marrow-derived MSCs exhibit better chondrogenesis than MSCs of adipose origin, but advantages such as high availability and high cell number makes adipose tissue a highly advantageous stem cell source to work with (Afizah et al., 2007).

\subsection{H\&E, alcian blue, safranin-0 and toluidine blue staining}

Chondrogenesis occurring in the hASCs laden hydrogels can be proved by the detection of GAGs deposition and proteoglycans protein production (Figure 4) with metachromatic staining for alcian blue, safranin $\mathrm{O}$ and toluidine blue. Figure 4 depicts the positive staining of hASCs encapsulated in $\kappa$-carrageenan for alcian blue, safranin-O and toluidine blue staining, demonstrating starting deposition of proteoglycans (GAGs), which is commonly found in native articular cartilage ECM. The staining with alcian blue (Figure 4A-D) indicated a concentration of acidic sulphated proteoglycans, and the cells appeared round within lacunae, which is a distinct morphological appearance and structural characteristic specific to cartilage. For safranin $\mathrm{O}$ staining, we could note the similarity in the intensity of the colour between the nucleus and cytoplasmic substance for the initial period of culture, and the difference in colour between these two for later days in culture (Figure 4E-H). An increase in the intensity of the staining that evolved from a more orthochromatic (blue) in the initial periods of culture to a more pronounced metachromatic (purple) staining in the later periods was noticed for toluidine blue staining (Figure 4I-L) similarly to mast cells found in connective tissue. This effect was more evident in the regions where cell clusters formed in comparison to individual cells present in the hydrogels. These results show that $\kappa$-carrageenan hydrogel is not only a lading system for the cell but is supporting the cells' functionality, namely the chondrogenic differentiation, mimicking ECM properties observed by the interaction and cell spreading within the hydrogel. By the end of the culture time, setting and partial chondrogenesis is taking place and a longer time in culture promises full differentiation of the hASCs. $\kappa$-Carrageenan hydrogels maintain the chondrocyte phenotype and promote cartilage-specific ECM deposition even without growth factor stimulation, as subsequently chondrogenic features were detected in cell-laden hydrogel cultured in basal medium, indicating that chondrogenic induction was hydrogel-dependent.

H\&E-stained sections (Figure 4M-P) show the typical tissue morphology; cell nuclei are stained dark blue while the cytoplasm and ECM have varying degrees of pink staining. At the end of week 1 of culture it is possible to observe a homogeneous distribution of cells with a smaller-sized basophilic nucleus (blue), while in the following weeks the cells assumed a more rounded morphology with cytoplasmic eosinophilic (pink) substance around the nucleus.

\subsection{Immunohistochemistry and real-time qRT-PCR}

To further evaluate the chondrogenic differentiation of hASCs encapsulated in 1.5\% w/v $\kappa$-carrageenan hydrogels, immunohistochemical analysis of specific proteins, such as types I and II collagen, was carried out. Figure 5A-D and E-H show that the cells were positive for collagen types II and I, respectively, during the studied culture times, and that the staining intensity increased with increasing length of culture, particularly for collagen type II. Differences were found at the histostructural level between the two proteins evaluated, with collagen type I being detected at early culture times, whereas positive staining for collagen type II was 


\section{Alcian Blue}
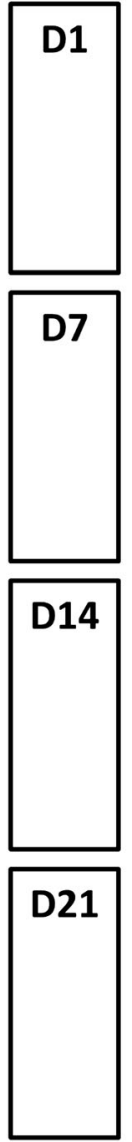

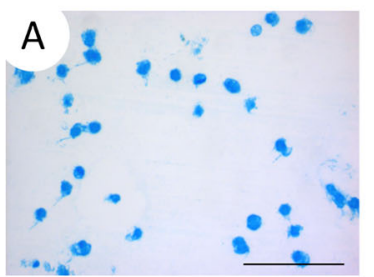

Safranin 0
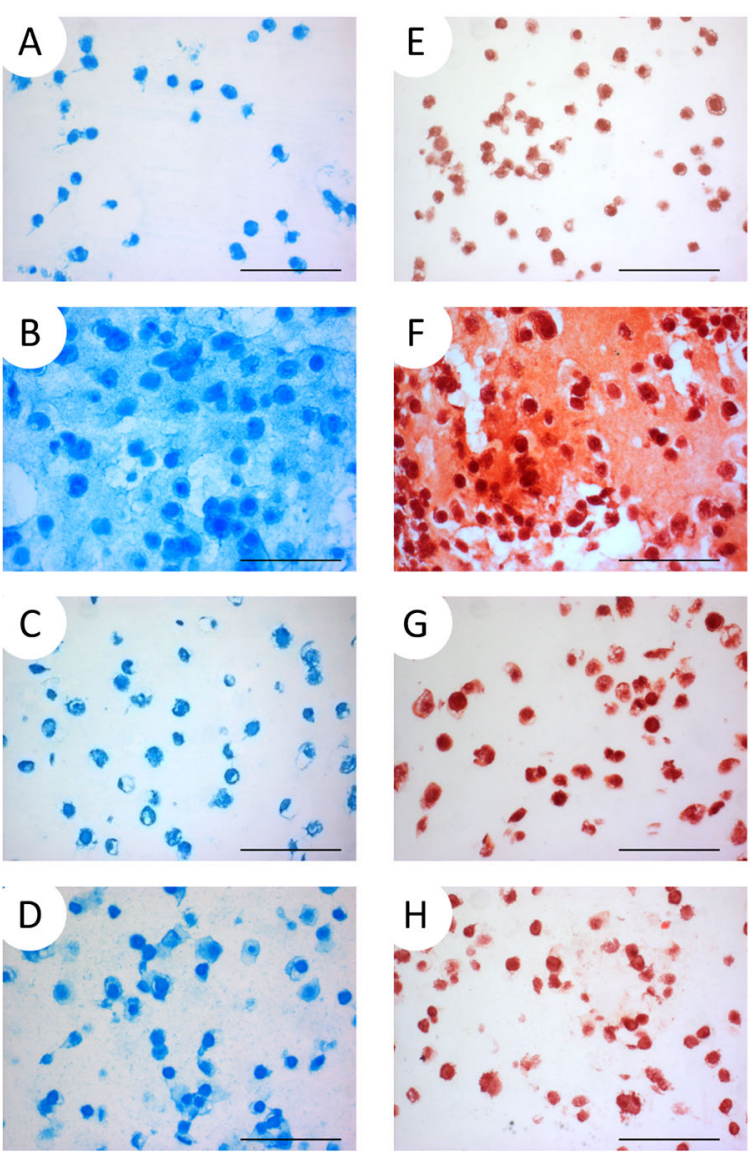
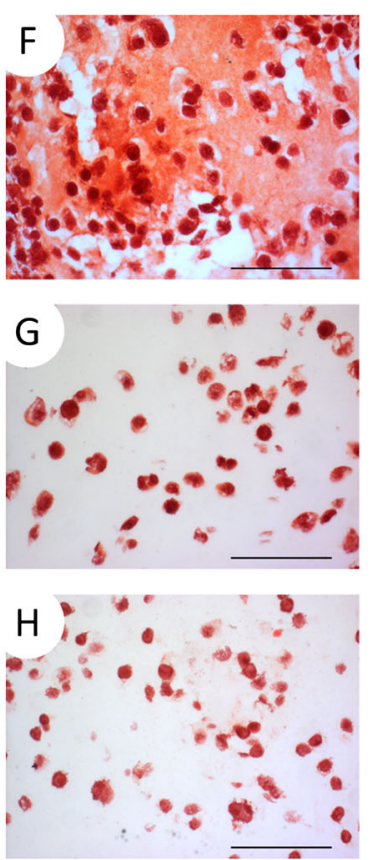

Toluidine Blue
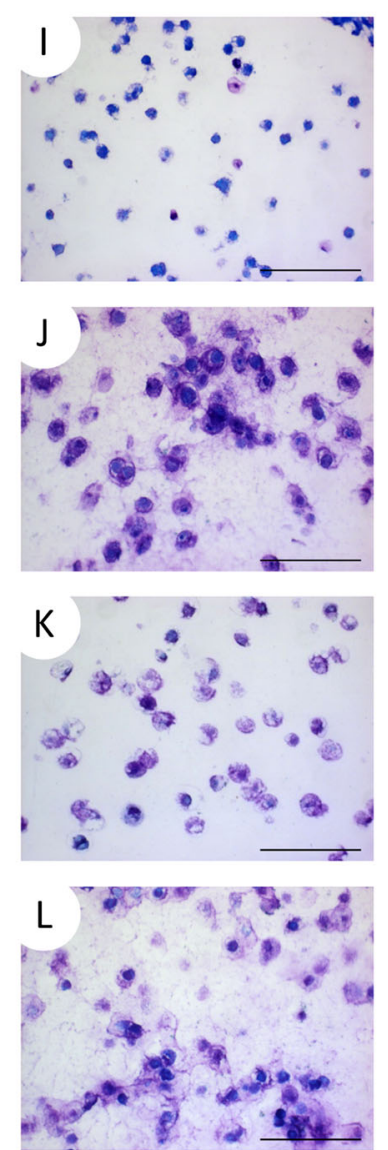

H\&E
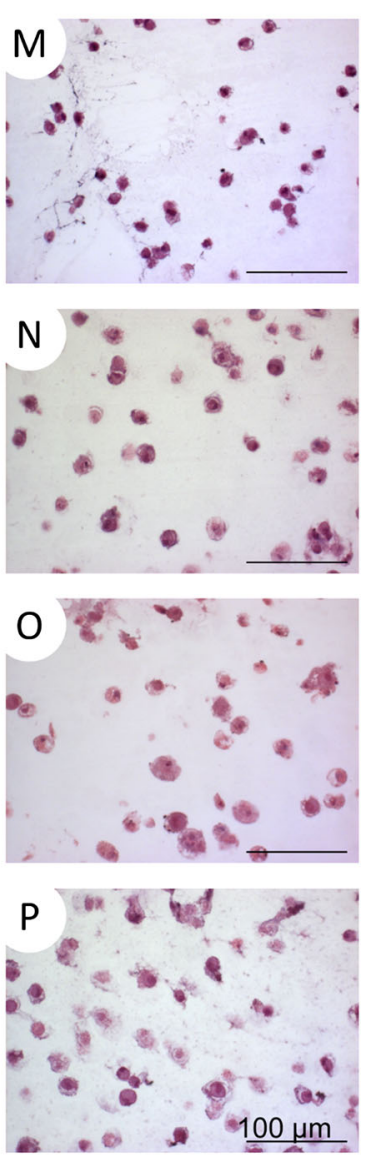

Figure 4. Optical microscopy images of histological sections obtained from $\kappa$-carrageenan hydrogels with encapsulated hASCs, collected after several periods of culture and stained with alcian blue (A-D), safranin O (E-H), toluidine blue (I-L) and H\&E (M-P). Scale bar $=100 \mu \mathrm{m}$; magnification $=\times 40$

detected for the latest culture times. However, chondrogenesis is typically a progressive process, from a morphogenetic phase to a cytodifferentiation phase of development via a number of precursor stages to the mature chondrocytes (Lin et al., 2006). Since a heterogeneous population was used, it was expected to also have positive staining for collagen type I. With time, the cells enlarged and secreted a dense organization around the nucleus. On close inspection, cells from 21 day culture were spherical in shape with cytoplasmic depositions. Although most of the cells had retained the round shape typically expressed by chondrocytes that populate the deeper layers of articular cartilage (Aydelotte and Kuettner, 1988), cells lying closer to the surface were flattened. No positive labelling was found in the negative control sections, given the absence of the key protein markers (Figure 5I-L). The presence of a metachromatic-staining matrix, the chondrocyte-like appearance of the cells and the detection of type II collagen suggest that the tissue generated by these cells is similar to native cartilage.

The chondrogenic differentiation of encapsulated hASCs was additionally evidenced by the results obtained from real-time qRT-PCR analysis, which allowed assessment of the expression, at a molecular level, of several important typical chondrogenic markers for hASCS encapsulated in $\kappa$-carrageenan hydrogels (Figure 6). Sustained gene expression levels were registered for Sox $9\left({ }^{*} p<0.05\right)$ and Aggrecan $\left({ }^{\#} p<0.05\right)$, increasing significantly with time from day 14 until the end of the experiment. Actually, Sox9 was upregulated to values close to 30 -fold from day 1 to day 21 . Collagen type II and Aggrecan are considered to be the two major and most important constituents of hyaline cartilage ECM, since the functionality of this tissue relies mostly on the presence of these components (Figure 6). Collagen type II gene expression decreased from day 7 to day 14 of culture, but was significantly increased from day 14 to day 21 $(* * p<0.05)$. The expression of Collagen type $I$, unlike Collagen type II, registered a two-fold increase between days 7 and 21 of culture $\left({ }^{\&} p<0.05\right)$. At the end of culture it was possible to notice that high mRNA expression of Sox9 remained, as well as gene expression of Aggrecan and of Collagen type II. It might be mentioned that Collagen type $X$, related to the hypertrophic stage of chondrogenic differentiation, registered a slightly increased expression over the culture time studied. In general, from the analysis of gene expression after 3 weeks of culture, different stages in the cell life cycle could be distinguished. Several studies reported in the literature (Mueller et al., 2010; Zscharnack et al., 2010), investigating the chondrogenic differentiation 

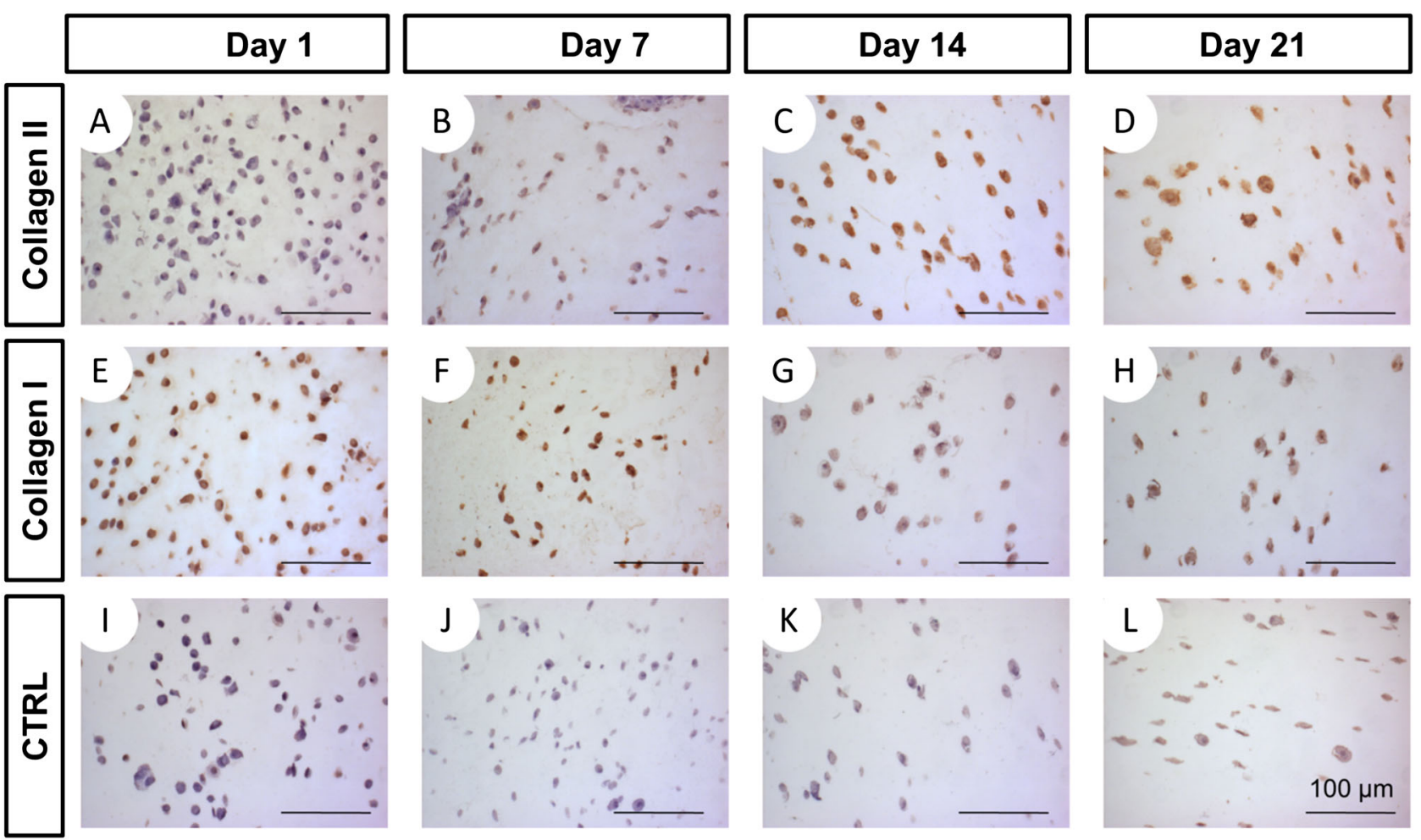

Figure 5. Light microscopy images obtained from immunohistochemical staining of hASCS encapsulated in $\kappa$-carrageenan hydrogels after 21 days of culture for collagen type II (A-D) and type I (E-H) and negative controls (I-L). Scale bar $=100 \mu \mathrm{m}$; magnification $=\times 40$

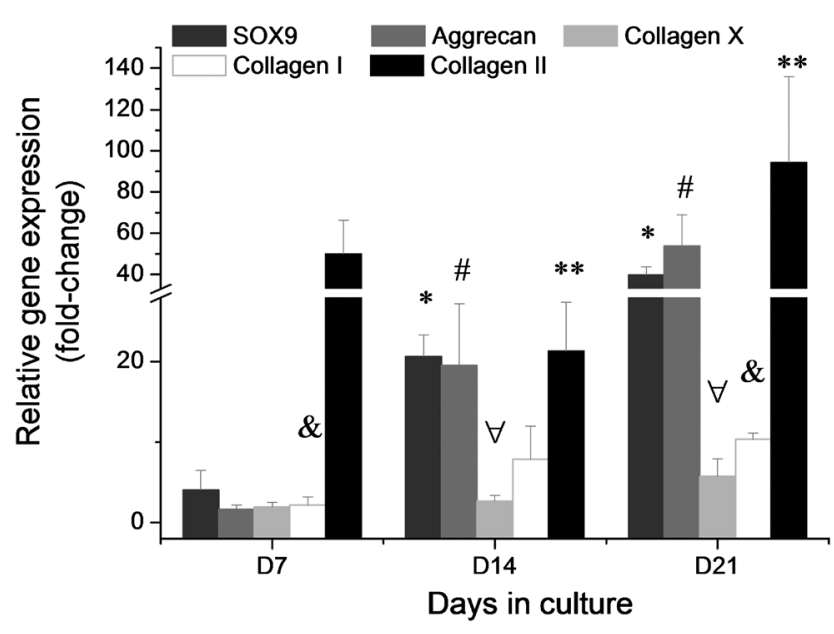

Figure 6. Relative expression of the chondrogenic specific genes SOX9, aggrecan, collagen X, collagen type I and collagen type II, based on the mRNA produced by encapsulated human ASCs after 7, 14 and 21 days of culture. The expression of these genes was normalized against the housekeeping gene GAPDH and calculated by the $\triangle C$ T method. The symbols denote statistically significant differences between day 14 and day 21 for the following genes: *, SOX9; **, collagen II; \#, aggrecan; $\forall$, collagen X; and the symbol \&, between day 7 and day 21 for collagen I gene. Statistical analyses were conducted using one-way ANOVA for $n=3 ; p<0.05$. Error bars represent mean $\pm S D$

of stem cells, state that chondrogenesis starts around day 14, when the cells start to express chondrogenic markers, as we also reported in this study. The time-frame of the study, namely 21 days of culture, was selected based on previous studies, with similar aims, using encapsulated stem cells in hydrogel for chondrogenic differentiation (Bosnakovski et al., 2006; Hwang et al., 2006; Zheng et al., 2009). Although it would be interesting to verify the outcomes in a long-term experiment, envisioning the clinical application of the proposed strategy, we were mostly concerned with the ability of the hydrogels to guarantee early predifferentiation, as a long-term in vitro culture before implantation is not interesting in a clinical/industrial scenario.

Sox9 gene expression was prematurely upregulated, which indicates the early onset of chondrogenesis (Ahmed et al., 2007). Sox9 is an important regulator of the chondrocyte phenotype (de Crombrugghe et al., 2000) and controls the expression of Collagen II, a well known marker of the ECM of cartilage, which is usually expressed in the end-stage of the chondrogenic process of differentiation (Goldring et al., 2006). In this system, hASCs were likely stimulated down the chondrogenic pathway by the growth factor TGF $\beta 1$ present in the chondrogenic medium. These findings indicated that hASCs can be successfully differentiated into chondrocytes when encapsulated in $\kappa$-carrageenan.

\subsection{Dynamic mechanical analysis (DMA)}

The compressive modulus is a particularly important parameter to consider in cartilage tissue engineering, as native cartilage is subjected to movement (i.e. walking, running); loading and unloading are transient events occurring in $<1 \mathrm{~s}$. Hence, the mechanical response of cartilage should be measured under dynamic cyclic conditions at a functionally relevant frequency, i.e. in the range 
0.5-3 Hz (Stolz et al., 2004; Ronken et al., 2012). The investigation of the viscoelastic properties of biodegradable hydrogel systems may be of great interest, because one can not only simulate the physiological dynamical loading but also access relevant fundamental information at the molecular level, from both a structural and a dynamic perspective (Oliveira et al., 2005). Moreover, because of its complex structure and interactions of its biochemical constituents, and due primarily to fluid flow through the solid matrix, cartilage behaves mechanically as a viscoelastic solid (Cohen et al., 1998).
DMA allowed the determination of the mechanical properties of $\kappa$-carrageenan hydrogels with encapsulated hASCs after different culture times (1, 7, 14 and 21 days) in either basal or chondrogenic medium (as compared to $\kappa$-carrageenan hydrogels without cells), while immersed in a PBS solution at $37^{\circ} \mathrm{C}$ and throughout a physiologically relevant range of frequencies. Storage (elastic) and loss (viscous) components of the complex modulus were determined and are presented in Figure 7. The storage modulus $\left(E^{\prime}\right)$ curve shows how the stiffness of the polymeric material changes with time in culture (Figure 7A).

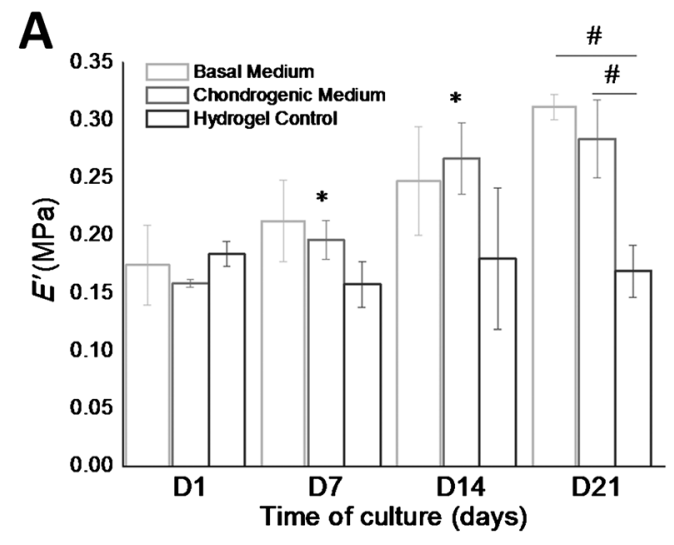

B

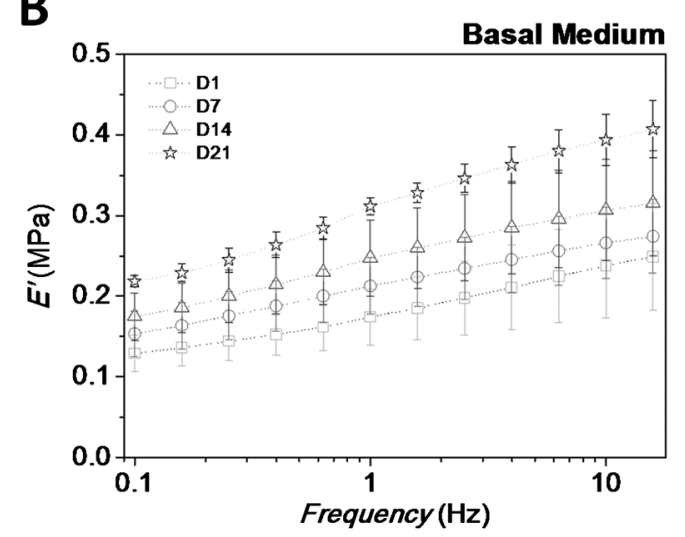

D

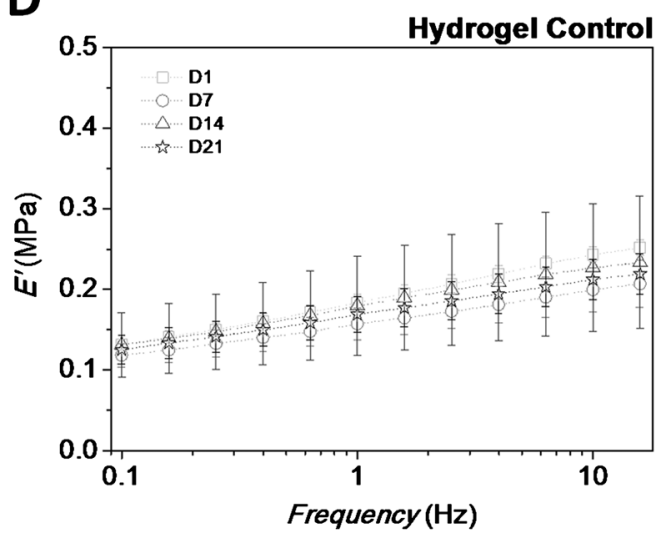

C

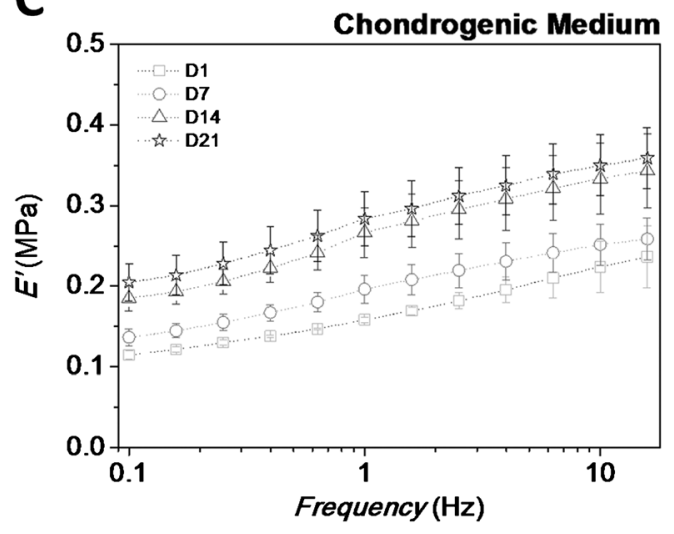

E

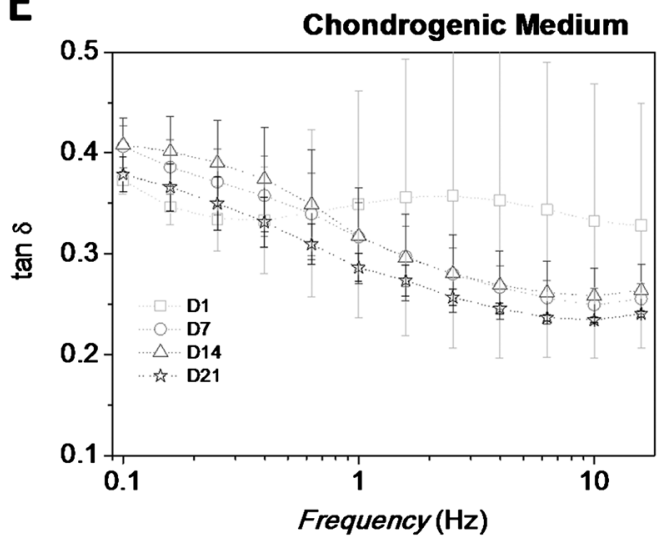

Figure 7. Storage modulus $\left(E^{\prime}\right)$ obtained from dynamic mechanical analysis upon compression of plain hydrogels and hydrogels with encapsulated hASCs, cultured in basal and chondrogenic media and tested at a frequency of $1 \mathrm{~Hz}$ (A). The frequency sweep test presents the storage modulus in a logarithmic scale at frequencies of $\mathbf{0 . 1 - 1 0 ~ H z ~ o f ~} \kappa$-carrageenan hydrogels with hASCs cultured in basal medium (B), hydrogels with hASCs exposed to chondrogenic medium (C) and hydrogels control (D). The damping factor $(\tan \delta)$ is displayed upon compression over a period of 21 days for chondrogenic condition $(E)$. Values reported correspond to an average of five test samples $(n=5) \pm$ SD 
At a frequency of $1 \mathrm{~Hz}$, the storage modulus of the hydrogels was estimated to be $0.23 \mathrm{MPa}$ for hASCs in basal medium, $0.22 \mathrm{MPa}$ for cells cultured in chondrogenic medium and $0.17 \mathrm{MPa}$ in $\kappa$-carrageenan gels alone by day 21 , revealing the elastic nature of these gels. In this study, the storage modulus $\left(E^{\prime}\right)$ and phase angle or damping $(\tan \delta$ ) were monitored as a function of frequency for the different hydrogels. The storage modulus curves show how the stiffness of the polymeric material changed with frequency (Figure 7B-D). Increase in $E^{\prime}$ was observed for increasing frequencies in the range $0.1-10 \mathrm{~Hz}$, which suggests that the hydrogels exhibited stiffer behaviour for higher frequencies. With the application of high frequencies to the hydrogel, the material became glassy and solid-like; at very low frequencies, the polymer exhibited a more liquid-like or rubbery response. The storage modulus of the hydrogel without cells presented variations between the initial and final time points, suggesting that the hydrogels become softer, possibly due to a loss of the cross-linking ions or to a loss in gel stability due to thermal factors, as it is a thermoreversible and ionic hydrogel. The elastic modulus of $\kappa$-carrageenan hydrogels was improved with encapsulation of hASCs and with increasing time in culture (Figure 7A). Similar behaviour was found for agarose or alginate hydrogels with the same type of cells and similar culture conditions (Awad et al., 2004). Nevertheless, $\kappa$-carrageenan with encapsulated hASCs exhibits a compression modulus approximately 10-fold higher than that reported for agarose and alginate hydrogels in the study by Awad et al. (2004). Hydrogels also possess damping capability that may be useful to dissipate cyclic mechanical energy that is imposed in an implantation scenario. 'Damping' is the term used for the general tendency of vibrating materials or structures to lose some elastic energy to internal heating or external friction; it tell us how good a material will be at absorbing energy (Mano et al., 2002). The corresponding graphs are shown in Figure 7E. An increase in $\tan \delta$ is observed from 0.1 to $10 \mathrm{~Hz}$ for day 1 for the chondrogenic medium condition, suggesting a higher dissipation capability of $\kappa$-carrageenan hydrogels at higher frequencies (Figure 7E). At the beginning of culture we could notice an increase in $\tan \delta$ values, but after 21 days in culture we could observe a decrease for increasing frequencies, which is related to the higher viscous component of hydrogel biomaterial.

During the 21 days in culture, DMA analysis indicated an increase in storage modulus and in the viscoelastic properties of $\kappa$-carrageenan gels with encapsulated cells, suggesting an increase in the stiffness possible due to ECM production. It is believed that the subsequent deposition of ECM components within the gel constructs was facilitated by the gradual degradation of the gels. At day 21 the statistical analysis indicated that the mean difference was significant at the 0.05 level between the cellencapsulating hydrogels compared to the cell-free hydrogel control. The mechanism behind the increase in stiffness of the cell-laden hydrogels likely involves more than the cell load, as it is a result of cell-mediated interactions and deposition of matrix with time. In addition, stem cells can proliferate as well as differentiate, but as cells differentiate their rate of replication usually decreases, and by the end of 21 days there is no difference in terms of elastic modulus values between cells exposed or not exposed to the differentiation medium. It has been demonstrated that the biomechanical properties of articular cartilage greatly depend on composition, density of ECM and interstitial fluid flow (water and solutes) (Evans and Quinn, 2005). Cell encapsulation and ECM deposition may therefore result in progressive increase of the mechanical properties of the 3D structures, as shown previously for the cell-hydrogel construct (Miyata et al., 2004).

The characterization at the molecular level correlated with the mechanical properties developed by these systems, as at day 14 we observed a decrease in the Collagen type II chondrogenic marker (responsible for the tensile properties); at the same time point there is an upregulation of Aggrecan gene expression (responsible for the compressive properties of the hydrogel), as these markers are interacting and are being expressed differently during chondrogenic differentiation. These values mimic the mechanical properties found in articular cartilage and they are higher or within the range of values found for other hydrogels used in similar cartilage-regenerative approaches (Kisiday et al., 2002; Nettles et al., 2004). Human articular cartilage possesses significant mechanical properties, with a compressive modulus of $0.79 \mathrm{MPa}$, a shear modulus of $0.69 \mathrm{MPa}$ and a tensile modulus of 0.3-10.2 MPa (Fisher et al., 2004). Evaluation of these properties contributed to a further understanding of the potential of these hydrogels for the target application.

Considering the suitable mechanical properties, together with the simplicity and reproducibility of the preparation process for $\kappa$-carrageenan hydrogel formation, which is performed under mild conditions without employing any extraneous toxic crosslinking agents, it is possible to conclude that such a matrix has potential applications in cartilage tissue-engineering applications. To further enhance the quality of engineered cartilage tissues formed from encapsulating hASCs within this system, one may optimize the release rates of supplemental growth factors incorporated in the hydrogels. It is also possible that host cells could be recruited to the gel, by release of the growth factors participating in cartilage formation.

\section{Conclusions}

This study presents $\kappa$-carrageenan as a potential hydrogel for cell delivery, with application in the regeneration of cartilage. In summary, the results obtained using $\kappa$-carrageenan hydrogels as a cell-loading matrix, prepared via an ionic crosslinking reaction, demonstrate that this system could be an alternative cell-delivery hydrogel system. hASCs encapsulated in $\kappa$-carrageenan 
hydrogels remain viable, proliferate and differentiate into the chondrogenic lineage. Furthermore, $\kappa$-carrageenan hydrogels with encapsulated hASCs were able to achieve mechanical properties, under compression, in the range of those reported for native cartilage. These promising in vitro results should be further analysed in animal model experiments designed to address fundamental questions concerning the in vivo degradation of the hydrogel and the possible in vivo differentiation of encapsulated cells.

\section{Conflict of interest}

The authors have declared that there is no conflict of interest.

\section{Acknowledgements}

Elena G. Popa would like to acknowledge the Portuguese Foundation for Science and Technology (PhD Grant No. SFRH/ $\mathrm{BD} / 64070 / 2009)$.

\section{References}

Afizah H, Yang Z, Hui JHP et al. 2007; A comparison between the chondrogenic potential of human bone marrow stem cells (BMSCs) and adipose-derived stem cells (ADSCs) taken from the same donors. Tissue Eng 13(4): 659-666.

Ahmed N, Dreier R, Gopferich A et al. 2007; Soluble signalling factors derived from differentiated cartilage tissue affect chondrogenic differentiation of rat adult marrow stromal cells. Cell Physiol Biochem 20(5): 665-678.

Augst AD, Kong HJ, Mooney DJ. 2006; Alginate hydrogels as biomaterials. Macromol Biosci 6(8): 623-633.

Awad HA, Quinn Wickham M, Leddy HA et al. 2004; Chondrogenic differentiation of adipose-derived adult stem cells in agarose, alginate, and gelatin scaffolds. Biomaterials 25(16): 3211-3222.

Aydelotte MB, Kuettner KE. 1988; Differences between sub-populations of cultured bovine articular chondrocytes. I. Morphology and cartilage matrix production. Connect Tissue Res 18(3): 205-222.

Baeza RI, Carp DJ, Pérez OE et al. 2002; $\kappa$-Carrageenan-protein interactions: effect of proteins on polysaccharide gelling and textural properties. Lebenson Wiss Technol 35(8): 741-747.

Bixler HJ. 1994; The carrageenan connection IV. Br Food $J$ 96(3): 12-17.

Bosnakovski D, Mizuno M, Kim G et al. 2006; Chondrogenic differentiation of bovine bone marrow mesenchymal stem cells (MSCs) in different hydrogels: influence of collagen type II extracellular matrix on MSC chondrogenesis. Biotechnol Bioeng 93(6): 1152-1163.

Carvalho PP, Wu X, Yu G et al. 2011a; The effect of storage time on adiposederived stem cell recovery from human lipoaspirates. Cells Tissues Organs 194(6): 494-500.

Carvalho PP, Wu X, Yu G et al. 2011b; Use of animal protein-free products for passaging adherent human adipose-derived stromal/ stem cells. Cytotherapy 13(5): 594-597.

Cohen NP, Foster RJ, Mow VC. 1998; Composition and dynamics of articular cartilage: structure, function, and maintaining healthy state. J Orthop Sports Phys Ther 28(4): 203-215.

de Crombrugghe B, Lefebvre V, Behringer RR et al. 2000; Transcriptional mechanisms of chondrocyte differentiation. Matrix Biol 19(5): 389-394.

Drury JL, Mooney DJ. 2003; Hydrogels for tissue engineering: scaffold design variables and applications. Biomaterials 24(24): 4337-4351.

Erickson GR, Gimble JM, Franklin DM et al. 2002; Chondrogenic potential of adipose tissue-derived stromal cells in vitro and in vivo. Biochem Biophys Res Commun 290(2): 763-769.

Evans RC, Quinn TM. 2005; Solute diffusivity correlates with mechanical properties and matrix density of compressed articular cartilage. Arch Biochem Biophys 442(1): 1-10.

Fisher JP, Jo S, Mikos AG et al. 2004; Thermoreversible hydrogel scaffolds for articular cartilage engineering. $J$ Biomed Mater Res A 71(2): 268-274.

Gimble JM, Katz AJ, Bunnell BA. 2007; Adipose-derived stem cells for regenerative medicine. Circ Res 100(9): 1249-1260.

Goldring MB, Tsuchimochi K, Ijiri K. 2006 The control of chondrogenesis. J Cell Biochem 97(1): 33-44.

Gomes ME, Reis RL, Cunha AM et al. 2001; Cytocompatibility and response of osteoblastic-like cells to starch-based polymers: effect of several additives and processing conditions. Biomaterials 22(13): 1911-1917.

Guilak F, Estes BT, Diekman BO et al. 2010; 2010 Nicolas Andry Award. Multipotent adult stem cells from adipose tissue for musculoskeletal tissue engineering. Clin Orthop Relat Res 468(9): 2530-2540.

Hwang NS, Varghese S, Zhang Z et al. 2006; Chondrogenic differentiation of human embryonic stem cell-derived cells in arginine-glycine-aspartate-modified hydrogels. Tissue Eng 12(9): 2695-2706.

Jen AC, Wake MC, Mikos AG. 1996; Review: hydrogels for cell immobilization. Biotechnol Bioeng 50(4): 357-364.

Jung Y, Chung YI, Kim SH et al. 2009; In situ chondrogenic differentiation of human adipose tissue-derived stem cells in a TGF $\beta$ 1-loaded fibrin-poly(lactide-caprolactone) nanoparticulate complex. Biomaterials 30(27): 4657-4664.

Kara S, Tamerler C, Bermek H et al. 2003; Cation effects on sol-gel and gel-sol phase transitions of $\kappa$-carrageenan-water system. Int J Biol Macromol 31(4-5): 177-185.

Kisiday J, Jin M, Kurz B et al. 2002; Selfassembling peptide hydrogel fosters chondrocyte extracellular matrix production and cell division: implications for cartilage tissue repair. Proc Natl Acad Sci USA 99(15): 9996-10001.

Kisiday JD, Kopesky PW, Evans $\mathrm{CH}$ et al. 2008; Evaluation of adult equine bone marrow- and adipose-derived progenitor cell chondrogenesis in hydrogel cultures. $J$ Orthop Res 26(3): 322-331.

Labarca C, Paigen K. 1980; A simple, rapid, and sensitive DNA assay procedure. Anal Biochem 102(2): 344-352.

Lennart P. 2006; Gelling carrageenans. In Food Polysaccharides and Their Applications. CRC Press: Boca Raton; 239-287.

Lin Z, Willers C, Xu J et al. 2006; The chondrocyte: biology and clinical application. Tissue Eng 12(7): 1971-1984.

Liski EP. 2009; Applied Statistics for Engineers and Physical Scientists, 3rd edn, by Johannes Ledolter, Robert V. Hogg. Int Stat Rev 77(3): 481-481.

Livak KJ, Schmittgen TD. 2001; Analysis of relative gene expression data using realtime quantitative PCR and the $2^{-\triangle C T}$ method. Methods 25(4): 402-408.

Mangione MR, Giacomazza D, Bulone D et al. 2005; $\mathrm{K}^{+}$and $\mathrm{Na}^{+}$effects on the gelation properties of $\kappa$-carrageenan. Biophys Chem 113(2): 129-135.

Mano JF, Reis RL, Cunha AM. 2002; Dynamic mechanical analysis in polymers for medical applications. In Polymer Based Systems in Tissue Engineering, Replacement and Regeneration, Reis RL, Cohn D (eds). Kluwer Academic: Dordrecht; 139-164.

Marsich E, Borgogna M, Donati I et al. 2008; Alginate/lactose-modified chitosan hydrogels: a bioactive biomaterial for chondrocyte encapsulation. $J$ Biomed Mater Res A 84A(2): 364-376.

Meunier V, Nicolai T, Durand D. 2001; Structure of aggregating $\kappa$-carrageenan fractions studied by light scattering. Int $J$ Biol Macromol 28(2): 157-165.

Miyata S, Furukawa KS, Ushida T et al. 2004; Static and dynamic mechanical properties of extracellular matrix synthesized by cultured chondrocytes. Mater Sci Eng C Mater Biol Appl 24(3): 425-429.

Mueller MB, Fischer M, Zellner $\mathrm{J}$ et al. 2010; Hypertrophy in mesenchymal stem cell chondrogenesis: effect of TGF- $\beta$ isoforms and chondrogenic conditioning. Cells Tissues Organs 192(3): 158-166.

Naim S, Samuel B, Chauhan B et al. 2004; Effect of potassium chloride and cationic drug on swelling, erosion and release from $\kappa$-carrageenan matrices. AAPS PharmSciTech 5(2): 25.

Nesic D, Whiteside R, Brittberg M et al. 2006; Cartilage tissue engineering for degenerative joint disease. Adv Drug Deliv Rev 58(2): 300-322.

Nettles DL, Vail TP, Morgan MT et al. 2004; Photo-crosslinkable hyaluronan as a scaffold 
for articular cartilage repair. Ann Biomed Eng 32(3): 391-397.

Oliveira AL, Mano JF, Román JS et al. 2005; Study of the influence of $\beta$-radiation on the properties and mineralization of different starch-based biomaterials. J Biomed Mater Res B Appl Biomater 74B(1): 560-569.

Prakash S, Martoni C. 2006; Toward a new generation of therapeutics: artificial cell targeted delivery of live cells for therapy. Appl Biochem Biotechnol 128(1): 1-22.

Rada T, Gomes ME, Reis RL. 2011; A novel method for the isolation of subpopulations of rat adipose stem cells with different proliferation and osteogenic differentiation potentials. J Tissue Eng Regen Med 5(8): 655-664.

Rada T, Reis R, Gomes M. 2010; Distinct stem cell subpopulations isolated from human adipose tissue exhibit different chondrogenic and osteogenic differentiation potential. Stem Cell Rev Rep 7(1): 64-76.

Redman SN, Oldfield SF, Archer CW. 2005; Current strategies for articular cartilage repair. Eur Cell Mater 9: 23-32; discussion, 23-32.

Ronken S, Arnold M, Ardura García H et al. 2012; A comparison of healthy human and swine articular cartilage dynamic indentation mechanics. Biomech Model Mechanobiol 11(5): 631-639.

Salgado AJ, Coutinho OP, Reis RL. 2004; Novel starch-based scaffolds for bone tissue engineering: cytotoxicity, cell culture, and protein expression. Tissue Eng 10(3-4): 465-474.

Shoichet MS, Li RH, White ML et al. 1996; Stability of hydrogels used in cell encapsulation: an in vitro comparison of alginate and agarose. Biotechnol Bioeng 50(4): 374-381.

Sipahigil O, Dortunc B. 2001; Preparation and in vitro evaluation of verapamil $\mathrm{HCl}$ and ibuprofen containing carrageenan beads. Int $J$ Pharm 228(1-2): 119-128.

Sittinger M, Hutmacher DW, Risbud MV. 2004; Current strategies for cell delivery in cartilage and bone regeneration. Curr Opin Biotechnol 15(5): 411-418.

Slaughter BV, Khurshid SS, Fisher OZ et al. 2009; Hydrogels in regenerative medicine. Adv Mater 21(32-33): 3307-3329.

Spagnuolo PA, Dalgleish DG, Goff HD et al. 2005; $\kappa$-Carrageenan interactions in systems containing casein micelles and polysaccharide stabilizers. Food Hydrocoll 19 (3): 371-377.

Stolz M, Raiteri R, Daniels AU et al. 2004; Dynamic elastic modulus of porcine articular cartilage determined at two different levels of tissue organization by indentation-type atomic force microscopy. Biophys J 86(5): 3269-3283.

Tapp H, Hanley EN Jr, Patt JC et al. 2009; Adipose-derived stem cells: characterization and current application in orthopaedic tissue repair. Exp Biol Med (Maywood) 234(1): 1-9.
Tuli R, Li WJ, Tuan RS. 2003; Current state of cartilage tissue engineering. Arthritis Res Ther 5(5): 235-238.

Vallee M, Cote JF, Fradette J. 2009; Adiposetissue engineering: taking advantage of the properties of human adipose-derived stem/stromal cells. Pathol Biol (Paris) 57(4): 309-317.

Varoni E, Tschon M, Palazzo B et al. 2012; Agarose gel as biomaterial or scaffold for implantation surgery: characterization, histological and histomorphometric study on soft tissue response. Connect Tissue Res 53(6): 548-554.

Velleman SG. 1999; The role of the extracellular matrix in skeletal muscle development. Poult Sci 78(5): 778-784.

Wei Y, Hu Y, Hao W et al. 2008; A novel injectable scaffold for cartilage tissue engineering using adipose-derived adult stem cells. J Orthop Res 26(1): 27-33.

Zheng L, Fan HS, Sun J et al. 2009; Chondrogenic differentiation of mesenchymal stem cells induced by collagen-based hydrogel: an in vivo study. $J$ Biomed Mater Res A 93A(2): 783-792.

Zscharnack M, Hepp P, Richter R et al. 2010; Repair of chronic osteochondral defects using predifferentiated mesenchymal stem cells in an ovine model. Am J Sports Med 38(9): 1857-1869.

Zuk PA, Zhu M, Ashjian P et al. 2002; Human adipose tissue is a source of multipotent stem cells. Mol Biol Cell 13(12): 4279-4295. 